\title{
Site-Specific Plan-View Sample Preparation in the FIB
}

\author{
S. McKernan, Y. Zhu, and J Xie
}

3M, 3M Center, St. Paul, MN55144.

Preparing site-specific cross-section TEM samples in the FIB or DualBeam instruments is fairly straightforward, particularly if the area of interest can be identified at the surface. It is particularly useful for samples composed of several different layers with different materials properties $[1,2]$. When the area of interest is not evident at the surface, or the area of interest is well below the surface and covers a reasonably large extended area a site-specific plan-view sample may be another option for the specimen geometry. In this paper we present a procedure for successfully producing planview samples from specific areas and at specific target depths below the surface of a sample - in the present case we demonstrate the procedure using a multilayer semiconductor sample.

Figure 1 shows some extended defects in a semiconductor structure that cross-section TEM imaging indicates are due to stacking fault pairs. In fig 2 we can see a plan view sample grown thin enough to be imaged directly in the TEM once the substrate was removed. It can be seen that only at the point of intersection of the faults do the two stacking faults come together. Cross-sections taken slightly above or below this point will show two separate stacking faults that originate above the interface plane, giving a misleading picture as to their origin. Figure 3 shows the site-specific plan-view image obtained by this technique from close to the interface responsible for forming the defect.

The remaining images represent different stages in the formation of the site-specific plan view specimen. Firstly the area of interest is converted into a mesa by milling trenches on all four sides (fig 4). One side of this mesa is then undercut with the ion beam at an angle of $52^{\circ}$ to the horizontal; the inclination of the ion beam to the electron beam (fig 5). The sample is then rotated $180^{\circ}$ to undercut the opposite side. Immediately prior to this second undercutting the Omniprobe needle is inserted and attached to the sample using the Pt deposition system (fig 6). After this second undercutting operation a wedge of material remains attached to the needle oriented with the area of interest normal to the electron beam. The remainder of the procedure consists in manipulating this wedge so that it is normal to the electron beam and can be bonded to a TEM grid with the Pt deposition system. This is accomplished by a 2-step process. First the wedge is welded to a TEM grid at $45^{\circ}$ to the electron beam (fig 7), and the Omniprobe needle cut free. The sample is then rotated $180^{\circ}$ so that the TEM grid is at $-45^{\circ}$ to the electron beam. At this point the surface of interest is now parallel to the electron beam. The wedge is again attached to the Omniprobe needle (fig 8) and cut free of the TEM grid. The TEM grid can now be re-oriented in the conventional manner parallel to the electron beam. The sample is $\mathrm{Pt}$ - bonded to it again and then freed from the Omniprobe needle for the final time. At this point the thinning and cleanup of the specimen is the same as for any other geometry.

For depth-specific plan view sections one can either make use of the fact that there is now a crosssection of the material uppermost and different materials may be discerned from their different secondary electron yield (fig 9) or one can simple measure back from the original surface to determine where to thin the section for TEM analysis.[3] 
References

[1] B. Schaffer et al. Ultramicroscopy 101 (2004) 123.

[2] P. Thangadurai et al. Materials Characterization 59 (2008) 1623

[3] The authors wish to thank Dr T. J. Miller for the semiconductor samples.
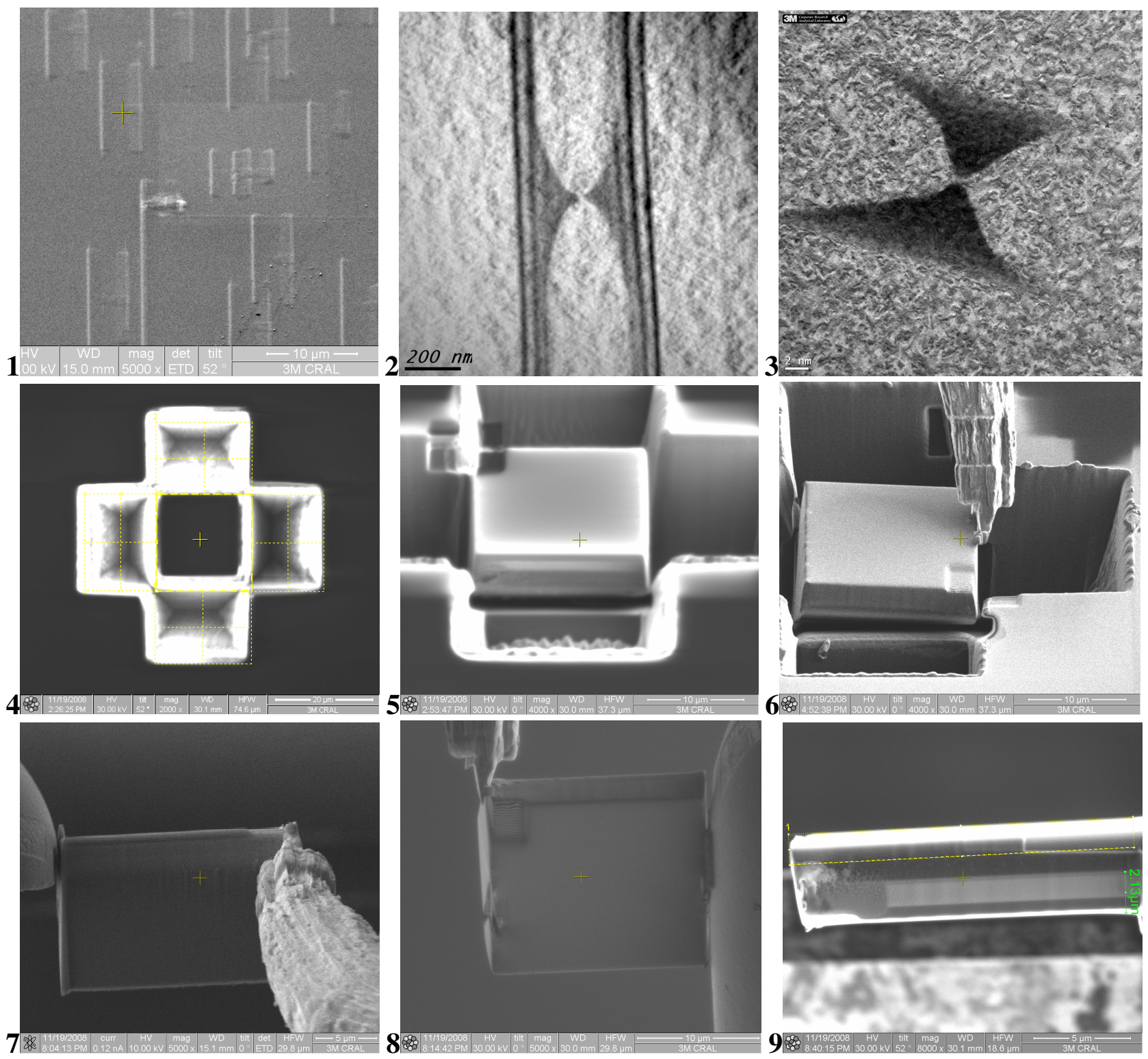

FIG. 1. SEM of extended defects in a compound II-VI semiconductor grown on InP.

FIG. 2. TEM image of the paired stacking-fault defect in a sample prepared by chemical etching. FIG. 3. TEM image of the defect in a TEM sample prepared using this procedure.

FIG. 4 - 9. Steps in the formation of a site-specific plan view specimen. See text for details. 